\title{
Qualidade do consumo alimentar e fatores associados em crianças de um ano de vida na Atenção Primária à Saúde
}

\author{
Dietary intake quality and associated factors in one year-old \\ children seen by primary healthcare services
}

Laura Garcia de Freitas (https://orcid.org/0000-0002-7658-479X) ${ }^{1}$

Margarita Alexandra Pena Cortés (https://orcid.org/0000-0003-2764-9152) ${ }^{2}$

Caroline Stein (https://orcid.org/0000-0003-4777-1630) ${ }^{3}$

Ewerton Cousin (https://orcid.org/0000-0003-3455-8865) ${ }^{4}$

Daniel Demétrio Faustino-Silva (https://orcid.org/0000-0001-6876-6537) ${ }^{5}$

Juliana Balbinot Hilgert (https://orcid.org/0000-0002-2204-1634) ${ }^{6}$
${ }^{1}$ Grupo Hospitalar

Conceição. Av. Francisco

Trein 596, Cristo Redentor

91350-200 Porto Alegre

RS Brasil.

laufreinutri@gmail.com

${ }^{2}$ Programa de Pós-

Graduação em Medicina e

Ciências da Saúde, Pontifícia

Universidade Católica do

Rio Grande do Sul. Porto

Alegre RS Brasil.

${ }^{3}$ Programa de Pós-

Graduação em Odontologia,

Universidade Federal do Rio

Grande do Sul (UFRGS).

Porto Alegre RS Brasil.

${ }^{4}$ Programa de Pós-

Graduação em

Epidemiologia, UFRGS.

Porto Alegre RS Brasil.

${ }^{5}$ Serviço de Saúde

Comunitária e Programa de

Pós-Graduação Modalidade

Mestrado Profissional

Avaliação e Produção

de Tecnologias para o

SUS, Grupo Hospitalar

Conceição. Porto Alegre RS

Brasil.

${ }^{6}$ Departamento de

Odontologia Preventiva

e Social, Faculdade de

Odontologia, UFRGS. Porto

Alegre RS Brasil.
Abstract The purpose of this paper was to analyze the food intake quality in one year-old children seen by a primary healthcare (PHC) service. This is a cross-sectional studied nested within a child oral health cohort study which collected data regarding children born in 2013 and monitored in Porto Alegre for two years. We applied a questionnaire on maternal variables and frequency of pediatric appointments, weight and height measurements, and children's food intake. To that end, a score was generated based on the points assigned according to SISVAN (meaning 'food and nutrition monitoring system,' run by the Brazilian Ministry of Health). A multivariate model was calculated using Poisson regression with robust variance. The sample comprised 249 children. We found $30.5 \%$ (76) of poor/regular dietary quality, which in the multivariate model was associated to the guardian's educational background, considering up to incomplete high school $(P R=2.14$, CI95\% $=1.03-4.44)$ and complete high school $(P R=1.70, C 195 \%=0.81-3.54)$, as well as their failure to see a dentist $(P R=2.54, C 195 \%=1.33$ 4.84) or having seen one before the age of four months $(P R=1.94$, CI95\% =1.01-3.72). It is our conclusion that failing to see a dentist within the first year of life and lower maternal schooling negatively impact on children's dietary quality.

Key words Child health, Dietary intake, Child care, Nutritional epidemiology
Resumo O objetivo foi analisar a qualidade do consumo alimentar de crianças com um ano de idade acompanhadas por um serviço de Atenção Primária à Saúde (APS). Trata-se de estudo transversal aninhado a uma coorte de saúde bucal infantil em que foram coletados dados de crianças nascidas em 2013 e acompanhadas por 2 anos, em Porto Alegre. Foi aplicado um questionário sobre variáveis maternas e frequência de consultas de puericultura, medidas antropométricas e consumo alimentar da criança. Para tal, foi gerado um escore a partir da pontuação criada conforme o SISVAN (Sistema de Vigilância Alimentar e Nutricional). Foi calculado um modelo multivariado, por meio da Regressão de Poisson com variância robusta. A amostra consistiu de 249 crianças. Encontrou-se 30,5\% (76) de qualidade ruim/regular da alimentação, que no modelo multivariado esteve associada com nível educacional do responsável, sendo até ensino médio incompleto $(R P=2,14$, $I C 95 \%=1,03-4,44)$ e ensino médio completo ( $R P$ $=1,70$, IC95\% = 0,81-3,54), assim como não ter consultado com dentista $(R P=2,54, I C 95 \%=$ 1,33-4,84) ou ter consultado até o quarto mês de idade $(R P=1,94$, IC95\% = 1,01-3,72). Conclui-se que não consultar com dentista no primeiro ano de vida e menor escolaridade materna repercutem negativamente na qualidade alimentar infantil.

Palavras-chave Saúde da criança, Consumo de alimentos, Puericultura, Epidemiologia nutricional 


\section{Introdução}

Conhecer os padrões alimentares no primeiro ano de vida pode ser estratégico para estabelecer ações de promoção e prevenção da saúde desde o nascimento até a vida adulta. Em 2016, 41 milhões de crianças menores de 5 anos apresentavam sobrepeso ou eram obesas, e, em 2025 esse problema pode atingir 70 milhões de crianças ${ }^{1,2}$. As Doenças Crônicas Não Transmissíveis (DCNTs) são a principal fonte de Carga Global de Doenças no Brasil estimando-se que $58 \%$ dos anos perdidos de vida precocemente se devem às DCNTs $^{3,4}$. Estes agravos em saúde estão relacionados, de alguma maneira, com o comportamento alimentar do indivíduo e uma vez que os hábitos alimentares são formados na infância, esse período seria o ideal para promover ações visando a práticas alimentares saudáveis ${ }^{5}$. Assim, é possível prevenir futuros desfechos clínicos insatisfatórios relacionados à má nutrição e ao excesso de peso, hoje considerados problemas de saúde pública ${ }^{6-8}$.

O Brasil por meio da Atenção Primária à Saúde (APS), coloca como uma de suas prioridades a promoção da alimentação saudável e a prestação de um acompanhamento contínuo e integral aos indivíduos e suas famílias em todos os ciclos de vida, incluindo a primeira infância ${ }^{3}$. Em 1999 foi aprovada a Política Nacional de Alimentação e Nutrição (PNAN) e um dos compromissos estabelecidos é a realização de forma contínua e sistemática o monitoramento da situação alimentar e nutricional da população brasileira9. Para tanto o Ministério da Saúde (MS) criou o Sistema de Vigilância Alimentar e Nutricional (SISVAN) com o intuito de ser uma ferramenta importante na vigilância alimentar e nutricional de todos os ciclos da vida, contribuindo para a formulação e a revisão de políticas públicas, identificando áreas geográficas, segmentos sociais e grupos populacionais de maior risco aos agravos nutricionais ${ }^{10}$.

A promoção e a recuperação da saúde infantil são prioridades do governo que através do serviço de puericultura oferecido na APS acompanha o crescimento e o desenvolvimento da criança prevenindo doenças na infância e também na vida adulta ${ }^{11-13}$. O trabalho interdisciplinar de uma equipe de profissionais da APS é relevante para a comunidade, uma vez que é através de estratégias de educação em saúde que se promove a saúde da criança e o empoderamento das mães proporcionando qualidade de vida às crianças ${ }^{14}$. Fatores socioeconômicos como o nível de escolaridade materno e a renda têm sido apontados como possíveis interferentes no processo de me- lhora na qualidade da alimentação da população. A relação entre escolaridade materna e qualidade alimentar está descrita na literatura e pode influenciar negativamente ou positivamente os hábitos alimentares das crianças ${ }^{15,16}$.

Conhecer o padrão alimentar no primeiro ano de vida é fundamental para o estabelecimento de condutas nutricionais adequadas visando ao desenvolvimento saudável das crianças e suas famílias, bem como ao planejamento de ações e programas de educação em saúde no contexto da Atenção Primária à Saúde. Diante disso, o objetivo deste estudo foi analisar a qualidade do consumo alimentar de crianças com um ano de idade acompanhadas por um serviço de Atenção Primária à Saúde e fatores associados.

\section{Métodos}

\section{Delineamento}

Trata-se de um estudo transversal aninhado a uma coorte de acompanhamento de saúde bucal infantil.

\section{Contexto}

O estudo foi realizado nas doze Unidades de Saúde pertencentes ao Serviço de Saúde Comunitária do Grupo Hospitalar Conceição (SSCGHC), localizadas na Zona Norte de Porto Alegre (RS), Brasil. É um serviço de Atenção Primária à Saúde, referência no atendimento do Sistema Único de Saúde (SUS), com 39 equipes de saúde da família que com seus processos de trabalho resolve, em média, 90 a $95 \%$ dos problemas de saúde das populações adscritas ${ }^{17}$. O programa de puericultura em que o presente estudo foi desenvolvido é realizado por profissionais da equipe mínima de saúde da família, do Núcleo de Apoio à Saúde da Família e da Residência Integrada com ênfase na Saúde da Família e Comunidade, compondo uma equipe interdisciplinar de médicos de família, dentistas, técnicos de saúde bucal, enfermeiros, técnicos e auxiliares de enfermagem, assistentes sociais, psicólogos, nutricionistas, farmacêuticos e residentes de diferentes áreas do saber, como nutrição, odontologia, psicologia, enfermagem, farmácia, medicina e assistência social.

\section{Participantes}

Este estudo faz parte de outro maior, cujo objetivo é avaliar os fatores associados à saúde na 
infância em um programa de saúde bucal voltado a crianças de 0 a 12 meses, na Atenção Primária à Saúde. Trata-se de uma coorte de nascidos vivos no ano de 2013 no território de abrangência do SSC-GHC. Para este estudo, especificamente, foram avaliados dados do primeiro segmento de 249 crianças com idade entre 12 e 24 meses e suas mães/responsáveis.

Os critérios de inclusão foram as crianças nascidas no ano de 2013 na área de abrangência das 12 UBSs do SSC-GHC e que estivessem regularmente cadastradas e em acompanhamento no Programa da Criança, além de mãe/pai/responsáveis considerado como responsável pelos cuidados de saúde bucal da criança e que acompanhava todas as consultas médicas e odontológicas na unidade de saúde. Os critérios de exclusão foram a criança ser portadora de necessidades especiais ou síndromes, as crianças que saíram do território de abrangência das unidades de saúde do SSCGHC por mais de 6 meses consecutivos e mães/ pais/responsáveis sem condições cognitivas de responder aos questionários do estudo. De acordo com o manual Atenção à saúde da criança de $0 \mathrm{a}$ 12 anos do Grupo Hospitalar Conceição, o acompanhamento ideal da puericultura prevê 7 consultas médicas no primeiro ano de vida, por ser um período adequado de oferta de imunizações e de orientações de promoção e prevenção, bem como uma consulta com o cirurgião-dentista.

\section{Amostra}

Os responsáveis e as crianças foram selecionados a partir de uma amostra probabilística das 12 Unidades de Saúde para o estudo da coorte. No ano de 2013, na área de abrangência do SSC-GHC, nasceram 674 crianças e, destas, 469 receberam a primeira consulta com o cirurgião dentista até os 12 meses de idade. Para este estudo incluímos as crianças de até 24 meses de idade, totalizando 249, que junto ao seu responsável eram usuários das Unidades de Saúde, tendo sido respondidos os questionários de consumo alimentar para esta faixa etária.

Foi realizado o cálculo de poder da amostra para análise de fatores associados, sendo que os parâmetros utilizados foram os seguintes: nível de confiança de $95 \%$, prevalência do desfecho (qualidade alimentar ruim/regular) no grupo não exposto variando entre $23 \%$ (quem ajuda a cuidar - pai) e $28 \%$ (Idade da criança - 12 meses ou menos), proporção exposto/não exposto entre 1:1 (sexo da criança) e 1:5 (idade da criança na primeira consulta), e razões de prevalência entre 1,7 (sexo da criança, número de filhos do responsável, acompanhamento de puericultura) e 2,0 (quem ajuda a cuidar da criança) encontrando-se um poder mínimo de $80 \%$. As variáveis de exposição utilizadas para os cálculos acima foram: sexo da criança, idade do responsável, escolaridade do responsável, número de filhos do responsável, situação conjugal, quem ajuda a cuidar da criança, criança vai a creche, e acompanhamento de puericultura.

Todos os responsáveis dos participantes concordaram em fazer parte da pesquisa e assinaram o Termo de Consentimento Livre e Esclarecido antes de sua inclusão no estudo. A pesquisa foi aprovada pelo Comitê de Ética e Pesquisa do Grupo Hospitalar Conceição.

\section{Variáveis}

\section{Maternas}

Foram coletados dados relacionados a escolaridade, idade e número de filhos. Além de renda familiar e percepção da mãe sobre a quantia da renda familiar ser suficiente. A frequência nas consultas de puericultura foi analisada através do sistema de informações do serviço de saúde.

\section{Criança}

A avaliação do consumo alimentar foi realizada através da aplicação de um questionário com a mãe/responsável da criança contendo as questões do Instrumento de marcadores do consumo alimentar para menores de dois anos do Sistema de Vigilância Alimentar e Nutricional (SISVAN) do Ministério da Saúde ${ }^{18}$. As variáveis estudadas foram o tempo de aleitamento materno exclusivo e total, a introdução de itens como açúcar, carne, vegetais e sulfato ferroso na alimentação. A aplicação dos questionários foi realizada por entrevistadores previamente treinados para a pesquisa e a coleta das medidas antropométricas por profissionais pertencentes às equipes de saúde.

$\mathrm{O}$ escore total derivado do instrumento do SIVAN foi composto pelas 15 questões, com variação de 0 a 17 pontos. Para fins de análise, e devido à ausência de um indicador qualitativo total do instrumento do SISVAN, foi utilizado um escore com classificação em quintis ${ }^{19}$. Desta forma, foi possível transformar o escore bruto em percentual usando a seguinte equação: escore padronizado $=($ escore bruto/17 $) \times 100$. Através do escore padronizado, dividiu-se a amostra em cinco grupos: (a) muito ruim $(\leq 20 \%)$; (b) ruim (21\%-40\%); (c) regular (41\%-60\%); (d) bom (61\%-80\%); (e) ótimo (> 80\%). Os escores cal- 
culados para as questões refletem, de forma indutiva, a qualidade da dieta e o consumo desses alimentos através da pontuação obtida.

Para aferir o peso e o comprimento das crianças foram utilizadas balanças pediátricas digitais (Indústrias Filizola S/S, balança modelo BP Baby) e antropômetros de diferentes marcas disponíveis nas unidades de saúde. O diagnóstico nutricional foi determinado pelas curvas da Organização Mundial da Saúde ${ }^{20}$ de IMC (Índice de Massa Corporal: peso/(comprimento ${ }^{2}$ ) e Estatura para Idade segundo faixa etária e sexo ${ }^{10}$.

\section{Análise estatística}

Foi realizada uma análise descritiva apresentando as frequências absolutas e relativas das variáveis categóricas, e mediana e intervalo interquartílico da variável numérica com distribuição assimétrica.

Para análise de fatores associados foi realizada análise bruta e ajustada através da regressão de Poisson com ajuste robusto de variância. A análise ajustada foi realizada pelo método de seleção para trás, de acordo com o modelo hierarquizado de análise, sendo as variáveis idade da criança (meses), sexo da criança e idade do responsável (anos) no nível 1 (para verificar o quanto uma mãe mais jovem ou mais velha/experiente ajuda ou piora o cuidado da criança, e se a idade e o sexo desta também está relacionado com a alimentação); escolaridade do responsável, número de filhos do responsável, situação conjugal, quem ajuda a cuidar da criança e se ela vai a creche no nível 2 (variáveis que tradicionalmente interferem na saúde da criança), e idade da criança (meses) na primeira consulta ao dentista, acompanhamento de puericultura e renda no nível 3 (variáveis de cuidado em saúde que podem estar relacionados entre si e que podem impactar na orientação e na implementação de uma alimentação saudável da criança). Dessa forma, a análise foi controlada para variáveis do mesmo nível e níveis acima, se mantendo no modelo aquelas com valor $\mathrm{p}<0,20$. Todas as análises estatísticas foram realizadas através do pacote estatístico Stata IC 13.1.

\section{Resultados}

Das 249 crianças avaliadas, 51,8\% (129) eram do sexo masculino e 77,1\% (192) tinham mais de 12 meses de idade. Apenas $28,9 \%$ (72) das crianças estudadas tiveram acompanhamento adequado de puericultura com o mínimo de sete consultas no primeiro ano, enquanto que das mães entrevistadas, $37,0 \%$ (92) tinham completado o ensino médio. Quanto a ir na creche, $72,7 \%$ (181) referiram que a criança não frequentava e 58,2\% (145) consultaram com dentista entre os cinco e doze meses de idade (Tabela 1).

Quanto ao consumo alimentar das crianças no primeiro ano de vida, ainda ocorrem inadequações, embora 39,8\% (99) das crianças entrevistadas tenham ficado em Aleitamento Materno Exclusivo (AME) até os 6 meses de idade, 16,1\% (40) ficaram em AME até os 4 meses e 16,2\% (39) ficaram em AME durante menos de 1 mês ou nunca foram amamentadas no peito. A maioria teve uma conduta correta com relação ao consumo de alimentos não recomendados antes de 6 meses de idade (Tabela 2). No entanto, $62,7 \%$ (163) e $51,8 \%$ (129) da amostra estudada consumiu, respectivamente, suco industrializado ou refresco em pó e refrigerante, no mês anterior à aplicação do questionário (Tabela 2). A classificação final do consumo alimentar por escore demonstrou que $30,5 \%$ da população estudada o obteve ruim/regular $(\leq 20 \%$ a $60 \%$ de qualidade alimentar).

No modelo hierarquizado de análise encontrou-se maior prevalência de qualidade ruim/ regular em crianças de responsáveis com menores níveis de escolaridade, sendo até ensino médio incompleto e ensino médio completo, 2,14 $($ IC95\% $=1,03-4,44)$ e $1,70($ IC95\% $=0,81-3,54)$ vezes maior a probabilidade, respectivamente, comparado com responsáveis com ensino superior ou mais. Além disso, também se encontrou maior prevalência de qualidade ruim/regular em crianças que não tiveram consulta com dentista ou tiveram até o oitavo mês do primeiro ano de vida, $2,54($ IC95\% $=1,33-4,84)$ e 1,65 IC95\% = $1,01-3,72)$ vezes maior a probabilidade, respectivamente, comparado com crianças que consultaram com dentista entre os nove e doze meses de idade (Tabela 3).

Em relação às características antropométricas, das crianças que tiveram qualidade alimentar ruim/regular, apresentaram $8,6 \%$ (5) e $13,8 \%$ (8), baixa e elevada classificação peso/idade. Quanto ao Îndice de Massa Corporal, 15,8\% (9) e $8,0 \%$ (11) das crianças eram obesas para qualidade alimentar ruim/regular e bom/ótimo, respectivamente. Em relação à classificação estatura/idade, 17,2\% (10) das crianças com adequação alimentar ruim/regular estavam com baixa estatura, enquanto que para bom/ótimo, 13,1\% (18) estavam com baixa estatura. Para estas associações, não houve o mesmo entre as variáveis. 
Tabela 1. Caracterização da amostra estudada em relação a variáveis sociodemográficas e puericultura, segundo responsável da criança, usuários das Unidades de Saúde pertencentes ao SSC-GHCa, de

Porto Alegre/Brasil, 2017. ( $\mathrm{N}=249)$.

\begin{tabular}{|c|c|c|}
\hline Variáveis & $\mathbf{N}$ & $\%$ \\
\hline \multicolumn{3}{|l|}{ Idade da criança (meses) } \\
\hline 12 ou menos & 57 & 22,9 \\
\hline Mais de 12 meses & 192 & 77,1 \\
\hline \multicolumn{3}{|l|}{ Sexo da criança } \\
\hline Masculino & 129 & 51,8 \\
\hline Feminino & 120 & 48,2 \\
\hline \multicolumn{3}{|l|}{ Idade do responsável (anos) } \\
\hline Menos de 25 & 71 & 28,5 \\
\hline $25-29$ & 64 & 25,7 \\
\hline $30-34$ & 62 & 24,9 \\
\hline 35 ou mais & 52 & 20,9 \\
\hline \multicolumn{3}{|l|}{ Escolaridade do responsável } \\
\hline $\begin{array}{l}\text { Até Ensino médio } \\
\text { incompleto }\end{array}$ & 119 & 47,7 \\
\hline Ensino médio completo & 92 & 37,0 \\
\hline Ensino superior ou mais & 38 & 15,3 \\
\hline \multicolumn{3}{|l|}{$\begin{array}{l}\text { Número de filhos do } \\
\text { responsável }\end{array}$} \\
\hline 1 & 133 & 53,4 \\
\hline 2 ou mais & 116 & 46,6 \\
\hline \multicolumn{3}{|l|}{ Situação conjugal } \\
\hline Solteira/Divorciada/Viúva & 64 & 25,7 \\
\hline Casada/Mora junto & 185 & 74,3 \\
\hline \multicolumn{3}{|l|}{ Quem ajuda a cuidar } \\
\hline Pai & 178 & 71,5 \\
\hline Avó & 71 & 28,5 \\
\hline \multicolumn{3}{|l|}{ Criança frequenta a creche } \\
\hline Não & 181 & 72,7 \\
\hline Sim & 68 & 27,3 \\
\hline \multicolumn{3}{|l|}{$\begin{array}{l}\text { Idade da criança (meses) } \\
\text { na primeira consulta ao } \\
\text { dentista }\end{array}$} \\
\hline Não consultou & 47 & 18,9 \\
\hline $1-4$ & 57 & 22,9 \\
\hline $5-8$ & 87 & 34,9 \\
\hline $9-12$ & 58 & 23,3 \\
\hline \multicolumn{3}{|l|}{$\begin{array}{l}\text { Acompanhamento de } \\
\text { puericultura da criança }\end{array}$} \\
\hline Não & 177 & 71,1 \\
\hline $\operatorname{Sim}$ & 72 & 28,9 \\
\hline $\begin{array}{l}\text { Renda }(\mathrm{R} \$ 1.000)-\text { md }(\mathrm{P} 25 \\
\text { - P75) }\end{array}$ & $\begin{array}{r}1,6(1- \\
2,5)\end{array}$ & \\
\hline \multicolumn{3}{|l|}{ Qualidade alimentar } \\
\hline $\begin{array}{l}\text { Ruim/ regular }(\leq 20 \% \text { a } \\
60 \%)\end{array}$ & 76 & 30,5 \\
\hline Boa/ ótima (61\% a > 80\%) & 173 & 69,5 \\
\hline
\end{tabular}

${ }^{a}$ Serviço de Saúde Comunitária do Grupo Hospitalar Conceição.

\section{Discussão}

O serviço de Atenção Primária à Saúde, no qual a amostra de mães e crianças estão inseridas, busca cumprir com os atributos essenciais da APS como primeiro contato, longitudinalidade, integralidade e coordenação ${ }^{21}$. Nesse contexto, há profissionais que realizam o pré-natal e que seguem acompanhando a família durante a puericultura, estabelecendo um vínculo entre equipe de saúde e família do recém-nascido ${ }^{22}$. Sobre o diagnóstico nutricional e padrão alimentar na faixa etária da população estudada, poucos estudos foram encontrados no Brasil, o que destaca o ineditismo da presente pesquisa e a importância de continuar estudando esta população específica.

A oferta de AME atingiu menos da metade da amostra estudada. Percentual semelhante ao encontrado na Pesquisa de Prevalência de Aleitamento Materno em Municípios Brasileiros, em que a prevalência de AME nos primeiros seis meses de vida foi de $41 \%{ }^{23}$. Em um estudo realizado na Bahia, com uma amostra de mães e crianças de até dois anos de idade, os pesquisadores propõem uma revisão das políticas públicas frente à interrupção do aleitamento materno e alertam para a necessidade de levar em consideração fatores ambientais sociodemográficos, antropométricos, biológicos e obstétricos ${ }^{24}$. Razão pela qual se faz importante a proximidade da equipe de saúde com as famílias assistidas no esclarecimento dos desafios do dia a dia relacionados à prática da amamentação.

A Política Nacional de Alimentação e Nutrição (PNAN) refere que a dieta dos brasileiros é formada pela combinação de alimentos tradicionais como arroz e feijão em conjunto com industrializados ultraprocessados como refrigerantes e doces, tendo o consumo aumentado a cada ano ${ }^{3}$. No presente estudo os resultados sobre o consumo de alimentos indutores de obesidade como o suco em pó, o refrigerante e os mingaus engrossados corroboram os resultados encontrados anteriormente por estudos que também avaliaram hábitos alimentares das crianças de zero a cinco anos de idade e relacionaram o consumo inadequado de carboidratos e gorduras em excesso à rotina familiar ${ }^{25}$, o que leva a crer que a orientação alimentar deve ser direcionada à família como um todo, dentro das suas possibilidades e realidades socioculturais.

Recente estudo avaliou a efetividade da Estratégia Nacional para Alimentação Complementar Saudável (ENPACS), de 340 crianças entre 6 e 12 
Tabela 2. Formulário de Marcadores e pontuação do escore de Consumo Alimentar - Crianças entre 6 meses e menos de 2 anos, segundo responsável da criança, usuários das Unidades de Saúde pertencentes ao SSC-GHCa, de Porto Alegre/Brasil, 2017. $(\mathrm{N}=249)$.

\begin{tabular}{|c|c|c|}
\hline Variáveis & Pontuação ${ }^{b}$ & $\mathrm{~N}(\%)$ \\
\hline \multicolumn{3}{|l|}{ A criança ontem recebeu leite de peito ${ }^{\star}$} \\
\hline Não & 0 & $123(49,4)$ \\
\hline Sim & 1 & $126(50,6)$ \\
\hline \multicolumn{3}{|l|}{ Se não, até que idade seu filho mamou no peito* } \\
\hline Nunca & 0 & $14(11,4)$ \\
\hline$<120$ dias & 1 & $40(32,5)$ \\
\hline$\geq 120$ dias & 2 & $69(56,1)$ \\
\hline \multicolumn{3}{|c|}{ Até que idade seu filho ficou em aleitamento materno exclusivo? } \\
\hline$<1$ mês ou nunca & 0 & $39(16,2)$ \\
\hline Até 1 mês & 0 & $11(4,4)$ \\
\hline Até 2 meses & 1 & $14(56)$ \\
\hline Até 3 meses & 1 & $20(8,0)$ \\
\hline Até 4 meses & 2 & $40(16,1)$ \\
\hline Até 5 meses & 2 & $26(10,4)$ \\
\hline Até 6 meses & 3 & $99(39,8)$ \\
\hline \multicolumn{3}{|l|}{ Ontem a criança comeu - $\mathrm{n}(\%)$} \\
\hline Verduras/legumes & 1 & $206(82,7)$ \\
\hline Fruta & 1 & $222(89,2)$ \\
\hline Carne & 1 & $205(82,3)$ \\
\hline Feijão & 1 & $208(83,5)$ \\
\hline Assistindo televisão & 0 & $69(27,7)$ \\
\hline Comida de panela & 1 & $218(87,6)$ \\
\hline Mingau com leite ou leite engrossado com farinha & 0 & $82(32,9)$ \\
\hline Sulfato Ferroso & 1 & $75(30,1)$ \\
\hline \multicolumn{3}{|c|}{ A criança recebeu mel/melado/açúcar/rapadura antes de 6 meses de idade? } \\
\hline Não & 1 & $200(80,3)$ \\
\hline Sim & 0 & $49(19,7)$ \\
\hline \multicolumn{3}{|c|}{$\begin{array}{l}\text { A criança recebeu papa salgada/comida de panela antes da idade } \\
\text { adequada?*}\end{array}$} \\
\hline Não & 1 & $139(55,8)$ \\
\hline Sim & 0 & $110(44,2)$ \\
\hline \multicolumn{3}{|c|}{ A criança tomou suco industrializado ou refresco em pó no último mês? } \\
\hline Não & 1 & $97(37,3)$ \\
\hline Sim & 0 & $163(62,7)$ \\
\hline \multicolumn{3}{|l|}{ A criança tomou refrigerante no último mês? } \\
\hline Não & 1 & $120(48,2)$ \\
\hline Sim & 0 & $129(51,8)$ \\
\hline
\end{tabular}

meses, e encontrou que houve um efeito positivo da estratégia, contribuindo para a redução do consumo de alimentos considerados não saudáveis, como alimentos ultraprocessados e bebidas como refrigerante e suco ${ }^{26}$. Estes achados relacionam-se à necessidade de expansão das práticas de estratégias e intervenções para uma alimentação saudável, visto que no presente estudo mais da metade das crianças haviam consumido suco industrializado ou refrigerante nas últimas 24 horas de aplicação do instrumento de avaliação.

Ao analisar as variáveis maternas, os resultados obtidos mostraram uma associação entre menores níveis de escolaridade e qualidade alimentar 
Tabela 3. Análise bruta e ajustada da associação das variáveis em estudo com a classificação do escore de qualidade alimentar ruim/regular, segundo responsável da criança, usuários das Unidades de Saúde pertencentes ao SSC-GHC, de Porto Alegre/Brasil, 2017. ( $\mathrm{N}=249)$.

\begin{tabular}{|c|c|c|c|c|c|}
\hline \multirow{2}{*}{ Variáveis } & \multirow{2}{*}{$\begin{array}{c}\text { Qualidade } \\
\text { alimentar } \\
\text { ruim/regular }\end{array}$} & \multicolumn{2}{|c|}{ Análise Bruta } & \multicolumn{2}{|c|}{ Análise Ajustada } \\
\hline & & RP (IC95\%) & $\mathbf{p}$ & RP (IC95\%) & $\mathbf{p}$ \\
\hline \multicolumn{6}{|l|}{ Nível 1} \\
\hline Idade da criança (meses) & & & 0,65 & & 0,61 \\
\hline 12 ou menos & 28,1 & 1 & & 1 & \\
\hline Mais de 12 meses & 31,3 & $1,11(0,70-1,77)$ & & $1,13(0,71-1,79)$ & \\
\hline Sexo da criança & & & 0,13 & & 0,14 \\
\hline Masculino & 34,9 & $1,35(0,92-1,98)$ & & $1,33(0,91-1,94)$ & \\
\hline Feminino & 25,8 & 1 & & 1 & \\
\hline Idade do responsável (anos) & & & 0,09 & & 0,10 \\
\hline Menos de 25 & 36,6 & $1,59(0,88-2,85)$ & & $1,57(0,88-2,80)$ & \\
\hline $25-29$ & 21,9 & $0,95(0,48-1,87)$ & & $0,94(0,48-1,86)$ & \\
\hline $30-34$ & 38,7 & $1,68(0,93-3,02)$ & & $1,66(0,92-2,99)$ & \\
\hline 35 ou mais & 23,1 & 1 & & 1 & \\
\hline \multicolumn{6}{|l|}{ Nível 2} \\
\hline Escolaridade do responsável & & & $0,05^{\star}$ & & $0,03^{*}$ \\
\hline Até Ensino médio incompleto & 35,3 & $1,92(0,94-3,91)$ & & $2,14(1,03-4,44)$ & \\
\hline Ensino médio completo & 29,4 & $1,59(0,76-3,35)$ & & $1,70(0,81-3,54)$ & \\
\hline Ensino superior ou mais & 18,4 & 1 & & 1 & \\
\hline Número de filhos do responsável & & & 0,08 & & 0,09 \\
\hline 1 & 35,3 & $1,41(0,96-2,09)$ & & $1,45(0,95-2,20)$ & \\
\hline 2 ou mais & 25,0 & 1 & & 1 & \\
\hline Situação conjugal & & & 0,08 & & 0,09 \\
\hline Solteira/ Divorciada/Viúva & 39,1 & $1,42(0,96-2,08)$ & & $1,39(0,95-2,05)$ & \\
\hline Casada/Mora junto & 27,6 & 1 & & 1 & \\
\hline Quem ajuda a cuidar & & & 0,09 & & 0,09 \\
\hline Pai & 33,7 & $1,50(0,93-2,41)$ & & $1,50(0,93-2,41)$ & \\
\hline Avó & 22,5 & 1 & & 1 & \\
\hline Criança vai a creche & & & 0,41 & & 0,49 \\
\hline Não & 32,0 & $1,22(0,77-0,90)$ & & $1,17(0,75-1,81)$ & \\
\hline Sim & 26,5 & 1 & & 1 & \\
\hline \multicolumn{6}{|l|}{ Nível 3} \\
\hline $\begin{array}{l}\text { Idade da criança (meses) na primeira } \\
\text { consulta ao dentista }\end{array}$ & & & 0,03 & & 0,04 \\
\hline Não consultou & 44,7 & $2,59(1,35-4,96)$ & & $2,54(1,33-4,84)$ & \\
\hline $1-4$ & 33,3 & $1,93(0,99-3,79)$ & & $1,94(1,01-3,72)$ & \\
\hline $5-8$ & 29,9 & $1,73(0,90-3,32)$ & & $1,65(0,87-3,10)$ & \\
\hline $9-12$ & 17,2 & 1 & & 1 & \\
\hline Acompanhamento de puericultura & & & 0,56 & & 0,47 \\
\hline Não & 31,6 & $1,14(0,74-1,75)$ & & $1,17(0,76-1,81)$ & \\
\hline Sim & 27,8 & 1 & & 1 & \\
\hline Renda (R\$1.000) - md (P25 - P75) & & $0,88(0,76-1,02)$ & 0,09 & $0,92(0,78-1,08)$ & 0,31 \\
\hline
\end{tabular}

ruim/regular nas crianças avaliadas. Este achado vai ao encontro de outro estudo, em que foi avaliada a qualidade da alimentação de 1.282 crianças de sete a dez anos do município de Vitória (ES) a partir de um indicador de itens alimentares da prática do desjejum. Aproximadamente $41 \%$ das 
crianças apresentaram alimentação de baixa qualidade, o que esteve associado com a baixa escolaridade materna e a ausência do pai no domicílio ${ }^{16}$. Por outro lado, um estudo com 1.371 pares de mães e crianças de zero a 35 meses de uma área rural, encontrou que ter ensino superior associouse tanto a maior adesão de práticas adequadas de alimentação recomendadas pelas Organização Mundial da Saúde (OMS), quanto para práticas inadequadas que incluíam lanches altamente processados e bebidas açucaradas, e que desta forma um nível educacional superior não demonstrou ser garantia de melhores práticas alimentares ${ }^{15}$. Em outro estudo com crianças entre 2 e 9 anos de idade, uma dieta com alto teor de gordura e açúcar esteve associada a menores níveis de educação dos pais, além disso também encontraram associados uma alimentação mais adequada com o aumento da escolaridade dos pais ${ }^{27}$.

Em relação ao monitoramento de puericultura realizado pelas equipes, apesar de não ter tido associação significativa com a qualidade alimentar neste trabalho, sabe-se que o acompanhamento do crescimento e do desenvolvimento da criança pelos serviços de saúde contribui para desfechos positivos em diversos aspectos. Um estudo exploratório sobre o cuidado da criança na prática de puericultura constatou que o enfermeiro se coloca como intermediário no processo de cuidado da criança e discutiu que apesar de a equipe de enfermagem ser a principal mediadora, outros profissionais como o nutricionista, o assistente social e o médico são necessários para complementar as condutas ${ }^{28}$.

No presente estudo considerou-se também a idade da criança na primeira consulta com o dentista, que no Serviço de Saúde Comunitária do GHC está incluída na puericultura com foco no cuidado integral e multiprofissional, sendo as orientações alimentares parte essencial da consulta odontológica tendo em vista a forte associação da alimentação com a cárie na primeira infância ${ }^{29}$. Sendo assim, as crianças que não consultaram ou que consultaram até o oitavo mês tiveram maior prevalência de qualidade alimentar ruim/regular comparadas com as que foram entre os 9 e 12 meses. Esse achado pode estar relacionado à transição alimentar que ocorre a partir dos quatro meses de idade (quando mães voltam a trabalhar após licença maternidade) e dos seis meses (fim do período de AME), podendo com 9 meses o(s) responsável(is) já estar(em) mais preparado(s) para uma melhor adequação alimentar do filho(a) e seguir as orientações dadas pela equipe de saúde. No entanto, é possível que exista colinearidade entre a variável consulta com o dentista e as socioeconômicas e por isso essa associação precisa ser vista com cautela e melhor explorada em estudos futuros.

Em relação às variáveis número de filhos, situação conjugal, quem ajuda a cuidar da criança e se ela vai a creche, mesmo não ocorrendo associação com qualidade ruim/regular da alimentação, considera-se que são fatores importantes para a avaliação alimentar da criança, sendo que o fato de no presente estudo não terem sido encontradas associações pode ser explicado pelo tamanho da amostra. Em relação à avaliação antropométrica é importante destacar que a mesma não foi realizada por um único avaliador, sendo uma limitação, que pode conter vieses de procedimentos mesmo que realizado treinamento prévio. Porém destaca-se que mesmo sendo este um estudo transversal, está aninhado a uma coorte de saúde infantil que pretende fazer observações e avaliações longitudinais futuras.

Fatores associados à pobreza, como saúde, nutrição e socioeconômicos, dificultam que crianças de países menos desenvolvidos alcancem o seu potencial de desenvolvimento ${ }^{30}$. Esta evidência vai ao encontro dos achados do presente estudo em que ao analisar as variáveis maternas, mães que possuíam menor nível de escolaridade apresentaram qualidade inferior de consumo alimentar para seus filhos. Considerando a melhora da escolaridade ser um desafio maior para os governos e sociedade, é importante que intervenções com incentivo e informações sobre o AME e boa qualidade alimentar se façam mais presentes nas políticas públicas de saúde. Um estudo de intervenção de prevenção da obesidade infantil sobre as práticas de alimentação, com 456 famílias hispânicas de baixa renda de crianças com média de idade 3,4 meses, o grupo de intervenção apresentou maior prevalência de aleitamento materno exclusivo e foi menos propenso a introduzir alimentos complementares e líquidos em comparação com os controles ${ }^{31}$.

É importante ressaltar que a maioria das pesquisas não está relacionada com a faixa etária estudada por estes autores, o que torna difícil uma comparação mais acurada e justifica o desenvolvimento de novas pesquisas no primeiro ano de vida das crianças. O presente estudo é relevante do ponto de vista dos achados através de métodos inovadores de pesquisa em serviço de atenção primaria em saúde, podendo servir de modelo para estudos futuros. Conclui-se que menor escolaridade materna e não consultar com dentista no primeiro ano de vida, repercutem ne- 
gativamente na qualidade alimentar infantil. É necessário ampliar a discussão da qualidade do consumo alimentar nos primeiros anos de vida e intensificar o monitoramento alimentar, antropométrico e a sensibilização familiar a partir dos serviços de Atenção Primária à Saúde.

\section{Colaboradores}

LG Freitas e DDF Silva participaram da concepção do projeto, coleta, análise, interpretação dos dados e redação do manuscrito. C Stein, E Cousin, MAP Cortés e JB Hilgert participaram da análise, interpretação dos dados, redação e revisão crítica relevante do conteúdo intelectual do manuscrito.

\section{Agradecimentos}

Ao Serviço de Saúde Comunitária do Grupo Hospitalar Conceição, pela disponibilidade e apoio para realização deste estudo, à equipe de pesquisa que aplicou os questionários e as mães que aceitaram respondê-los. Ao Centro de Pesquisa em Odontologia Social (UFRGS). À Fundação de Amparo à Pesquisa do Estado do Rio Grande do Sul. Ao Conselho Nacional de Desenvolvimento Científico e Tecnológico. A Coordenação de Aperfeiçoamento de Pessoal de Nível Superior - Brasil (CAPES) - Código de Financiamento 001.

\section{Referências}

1. World Health Organization (WHO). Facts and figures on childhood obesity. Geneva: WHO; 2017.

2. World Health Organization (WHO). Obesity and overweight. Geneva: WHO; 2016.

3. Brasil. Ministério da Saúde (MS). Política Nacional de Alimentação e Nutrição. Brasília: MS; 2013.

4. Schmidt MI, Duncan BB, Silva GA, Menezes AM, Monteiro CA, Barreto SM, Chor D, Menezes PR. Chronic non-communicable diseases in Brazil: burden and current challenges. Lancet 2011; 377(9781):1949-1961.

5. Laws R, Campbell K, Pligt P, Ball K, Lynch J, Russell G, Taylor R, Denney-Wilson E. Obesity prevention in early life: an opportunity to better support the role of Maternal and Child Health Nurses in Australia. BMC Nursing 2015; 14:26.

6. Helle C, Hillesund ER, Omholt ML, Øverby NC. Early food for future health: a randomized controlled trial evaluating the effect of an eHealth intervention aiming to promote healthy food habits from early childhood. BMC Public Health. 2017; 17:729.

7. Brasil. Ministério da Saúde (MS). Guia alimentar para a população brasileira. Brasília: MS; 2014.

8. Daniels LA, Mallan KM, Nicholson JM, Thorpe K, Nambiar S, Mauch CE, Magarey A. An Early Feeding Practices Intervention for Obesity Prevention. Pediatrics 2015; 136(1):e40-e49.

9. Brasil. Ministério da Saúde (MS). Indicadores de Vigilância Alimentar e Nutricional: Brasil 2006. Brasília: MS; 2009.

10. Brasil. Ministério da Saúde (MS). SISVAN: Orientações básicas para coleta, o processamento, a análise de dados e a informação em serviços de saúde. Brasília: MS; 2011.

11. Santos AS, Duro SMS, Cade NV, Facchini LA, Tomasi E. Quality of infant care in primary health services in Southern and Northeastern Brazil. Rev Saude Publica 2018; 52:11. 
12. Gauterio DP, Irala DA, Cezar-Vaz MR. Puericultura em Enfermagem: perfil e principais problemas encontrados em crianças menores de um ano. Rev Bras Enferm 2012; 65(3):508-513.

13. Brasil. Ministério da Saúde (MS). Agenda de compromissos para a saúde integral da criança e redução da mortalidade infantil. Brasília: MS; 2004.

14. Vasconcelos VM, Frota MA, Martins MC, Machado MMT. Puericultura em enfermagem e educação em saúde: percepção de mães na estratégia saúde da família. Esc Anna Nery 2012; 16(2):326-331.

15. Contreras M, Blandón EZ, Persson LA, Hjern A, Ekström EC. Socio-economic resources, young child feeding practices, consumption of highly processed snacks and sugar-sweetened beverages: a population -based survey in rural northwestern Nicaragua. $B M C$ public health 2015; 15:25.

16. Molina MCB, Lopéz PM, Faria CP, Cade NV, Zandonade E. Socioeconomic predictors of child diet quality. Rev Saude Publica 2010; 44(5):785-732.

17. Grupo Hospitalar Conceição (GHC). Serviço de Saúde Comunitária Porto Alegre: Grupo Hospitalar Conceição; 2017 [acessado 2017 abr 26]. Disponível em: https://www.ghc.com.br/default.asp?idMenu=unidades\&idSubMenu $=5$

18. Brasil. Ministério da Saúde (MS). Informe: Uso dos formulários e registro das informações no novo Sistema Informatizado da Vigilância Alimentar e Nutricional Sisvan Web. Brasília: MS; 2008.

19. Brasil. Ministério da Saúde (MS). Dez passos para uma alimentação saudável: guia alimentar para crianças menores de dois anos. Brasília: MS; 2010.

20. Organización Mundial de la Salud (OMS). Curso de capacitación sobre la evaluación del crecimiento del niño: versión 1. Geneva: OMS; 2006.

21. Starfield B. Primary Care: balancing health needs, services, and technology. New York: Oxford University Press; 2002.

22. Brasil. Ministério da Saúde (MS). Grupo Hospitalar Conceição. Gerência de Saúde Comunitária. Atenção à saúde da criança de 0 a 12 anos. Porto Alegre: Hospital Nossa Senhora da Conceição; 2009.

23. Brasil. Ministério da Saúde (MS). Pesquisa de Prevalência de Aleitamento Materno em Municípios Brasileiros: Situação do Aleitamento Materno em 227 municípios brasileiros. Brasília: MS; 2010.

24. Demétrio F, Pinto EJ, Assis AMO. Fatores associados à interrupção precoce do aleitamento materno: um estudo de coorte de nascimento em dois municípios do Recôncavo da Bahia, Brasil. Cad Saude Publica 2012; 28(4):641-654.
25. Silva VP, Zurita RCM. Prevalência dos Fatores de Risco da Obesidade Infantil nos Centros Municipais de Educação Infantil do Município de Maringá-PR-2010. Saúde e Pesquisa 2012; 5(1):9-25.

26. Baldissera R, Issler RMS, Giugliani ERJ. Efetividade da Estratégia Nacional para Alimentação Complementar Saudável na melhoria da alimentação complementar de lactentes em um município do Sul do Brasil. Cad Saude Publica 2016; 32(9):e00101315.

27. Fernández-Alvira J, Mouratidou T, Bammann K, Hebestreit A, Barba G, Sieri S, Reisch L, Eiben G, Hadjigeorgiou C, Kovacs E, Huybrechts I, Moreno LA. Parental education and frequency of food consumption in European children: The IDEFICS study. Public Health Nutr 2013; 16(3):487-498.

28. Andrade RD, Santos JS, Pina JC, Silva MAI, Mello DF. A puericultura como momento de defesa do direito à saúde da criança. Ciência, Cuidado e Saúde 2013; 12(4):719-727.

29. Machado APS, Brunetto S, Faustino-Silva DD. Relato de Experiência de Atendimento Conjunto entre Odontologia e Nutrição a Crianças de 0 a 36 Meses em uma Unidade Básica de Saúde no Município de Porto Alegre-RS. Rev Fac Odontol 2011; 52(1/3):49-55.

30. Walker SP, Wachs TD, Grantham-McGregor S, Black MM, Nelson CA, Huffman SL, Baker-Henningham H, Chang SM, Hamadani JD, Lozoff B, Gardner JM, Powell CA, Rahman A, Richter L. Inequality in early childhood: risk and protective factors for early child development. Lancet 2011; 378(9799):1325-1338.

31. Gross RS, Mendelsohn AL, Gross MB, Scheinmann R, Messito MJ. Randomized Controlled Trial of a Primary Care-Based Child Obesity Prevention Intervention on Infant Feeding Practices. J Pediatr 2016; 174:171-177.

Artigo apresentado em 18/09/2017

Aprovado em 25/09/2018

Versão final apresentada em 30/09/2018 